\title{
Osseous metaplasia of the cervix: A rare transformation
}

\author{
Eman Elkattan ${ }^{1}$, Mona Abdelbadei ${ }^{1}$, Hatem Abdelmoaty ${ }^{1}$, Eman Ali ${ }^{1}$, Dalia Samir ${ }^{1}$, Hala Kheidr ${ }^{2}$ \\ 'Department of Obstetrics and Gynecology, Cairo University, Cairo, Egypt \\ ${ }^{2}$ Department of Pathology, Cairo University, Cairo, Egypt
}

\section{Abstract}

\begin{abstract}
Although numerous cases of endometrial osseous metaplasia appear in the literature, only 6 cases of osseous metaplasia of the cervix have been reported since 1982.A 30 years old nulligravida women was referred to our colposcopy clinic with cervical mass. General, gynecologic and colposcopic assessments were done. The patient had an excision biopsy. Diagnosis of cervical osseous metaplasia was confirmed on histologic examination.The cervical epithelium has the potency to differentiate to multiple types of epithelium including osseous epithelium.
\end{abstract}

(J Turk Ger Gynecol Assoc 2015; 16: 58-9)

Keywords: Osseous metaplasia, cervix, colposcopy

Received: 24 July, 2013

Accepted: 17 October, 2013

\section{Introduction}

Cervical metaplasia represents the replace of the endocervical epithelium with tubal, intestinal, squamous, endometrial, transitional, cartilaginous or osseous (1).

We are presenting a case of osseous metaplasia of the cervix which is a rare pathological transformation. The etiology of osseous metaplasia has given rise to controversies; various theories of the uterine bone formation have been discussed $(2,3)$.

Some studies showed that osseous metaplasia might be generated from stromal tissue by differentiation of fibroblast into osteoblastes. Other studies hypothesized that it is secondary to abortion and retention of fetal bone, a tissue that may initiate osteogenesis (4-6).

Most patients having osseous metaplasia present with menstrual irregularities, pelvic pain, bloody vaginal discharge, infertility and sterility (7).

\section{Case Presentation}

30 years-old nulligravida woman was referred to our colposcopy service because of an incidental finding of cervical mass whilst investigating infertility. She had a history of primary infertility for 2 years. She was also complaining of postcoital, contact bleeding and dyspareunia for 1.5 year. The patient was non-smoker. She denied any previous history of cervical or uterine trauma. The patient was medically free. She had no history of previous surgeries. General and abdominal examination revealed no significant findings. Inspection of the vulva revealed no abnormality. On speculum examination: a cervical whitish solid mass was noted. It was approximately
$2.5 \mathrm{~cm} \times 1.5 \mathrm{~cm}$ on the Anterior lip of cervix. A smear was taken. A high vaginal swab and ectocervical and endocervical smears were taken as well. On palpation, the cervix was hard as well as the mass. Colposcopic examination was performed (Zeiss, Germany) (Figure 1). On applying the acetic acid, no acetowhite changes were noted. Taking a punch biopsy was attempted but unfortunately it failed due to the hardness of the mass. The patient had an excisional biopsy in the theatre under regional anaesthesia. The smear result showed no abnormality. The vaginal and endocervical swaps revealed no evidence of infection. Microscopic examination of the excised cervical mass showed mature bone tissue of metaplastic origin (Figure 2).

Blood tests were taken to exclude metabolic disorders that can lead to calcification such as hypercalcemia, hypervitaminosis D, and hyperphosphatemia. Hysteroscopy and endometrial sampling were performed and revealed no abnormality. Informed consent was obtained.

\section{Discussion}

Osseous metaplasia (OM) is defined by the presence of heterotopic normal bone tissue in a soft tissue (8).

Overall, osseous metaplasia occurs in approximately 0.3 per 1000 women (9). The pathogenesis is still not clear. Inflammatory and traumatic processes had been incriminated. Injury to the cervix either by surgical trauma or by recurrent infection may cause the mesenchymal cells to undergo osseous metaplasia (9).

The women with osteoid metaplasia may be presented with dyspareunia, postcoital bleeding, infertility and leucorrhea $(6,10)$. Osteoid metaplasia of the uterus is a rare disease that affects the uterus and was reported after abortion, in chronic endo- 


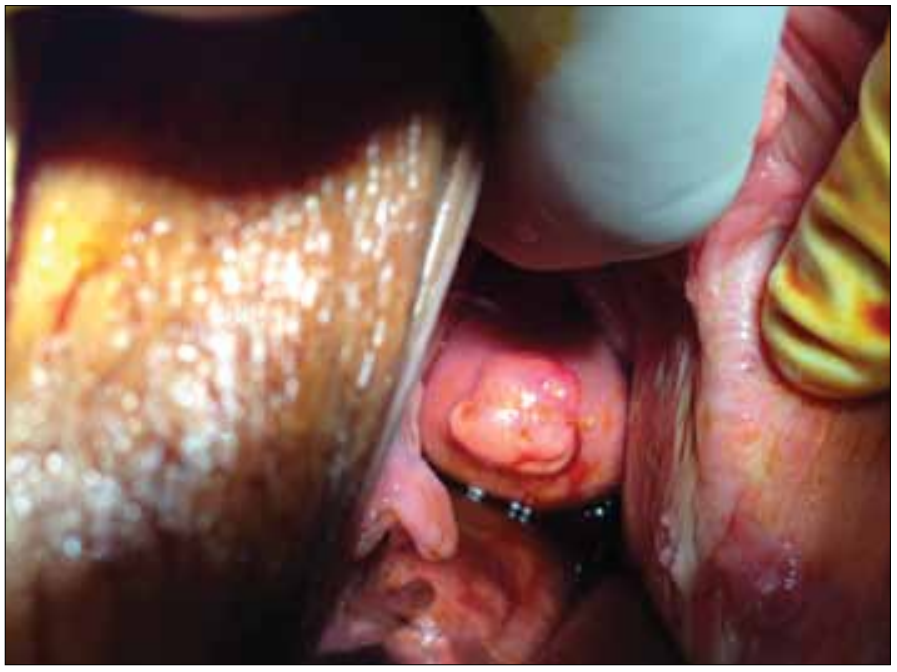

Figure 1. Shows the colposcopic examination of the cervical mass

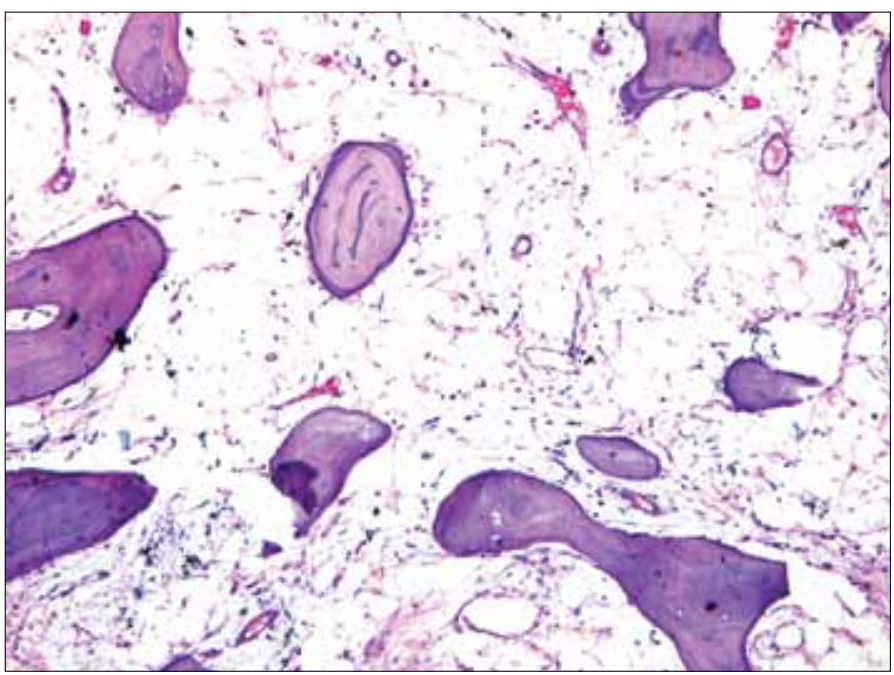

Figure 2. Shows the histopathology of the cervical mass after excision

metritis, metabolic disorders, and electro excision of the cervix (e.g. for severe cervical dysplasia). Retention of the fetal parts should be excluded. Bedaiwn et al. (4) reported a case of a woman that developed osseous metaplasia of the cervix shortly after loop electrosurgical excision procedure (LEEP) for severe cervical dysplasia. In this case the bone formation rapidly recurred after initial removal (4). There were previous 6 reported cases of Osseous metaplasia of the cervix in the literature. Physicians investigated the endometrium in only 3 of 6 cases, and in 2 of these, concomitant endometrial osseous metaplasia was found. In these 2 patients, chronic inflammation secondary to tissue damage was thought to be the principal cause. Therefore, because cervical osseous metaplasia can be associated with endometrial metaplasia, an evaluation of the endometrial cavity would be useful $(11,12)$.

We are reporting a very rare case of osseous metaplasia of the cervix. The aetiopathogenesis remains unclear. Although there was no concomitant osseous metaplasia in the uterine cavity, it may worth examining its effect on potential fertility.

Ethics Committee Approval: Ethics committee approval was received for this study from the ethics committee of Cairo University.

Informed Consent: Written informed consent was obtained from patients who participated in this study.

Peer-review: Externally peer-reviewed.

Author contributions: Concept - E.E., M.A.; Design - E.E., M.A.; Supervision - M.A.; Resource - H.A., E.A., D.S., H.K.; Materials - E.E., H.A., E.A., D.S., H.K.; Data Collection\&/or Processing - E.E.; Analysis\&/ or Interpretation - E.A., D.S.; Literature Search - E.E., H.A., E.A.; Writing - E.E., H.A., E.A.; Critical Reviews - E.E., H.A., E.A.

\section{References}

1. Simionescu C, Margaritescu C, Georgescu V, Mogoanta L, Marinescu AM. Pseudo-tumoral lesions of the cervix. Rom J Morphol Embryol 2005; 46: 239-247.

2. Duguid JP. Microbiol. Infections Churchill Livingstone, 1978; p. 304.

3. Niccoli V, Carillo C, Caruso MT, Lippa A, Serrao L. Clinicaldiagnostic considerationes of a case of endometrial ossification as a causeof secundary infertility. Minerva Gynecol 1997; 49: 289-91.

4. Bedaiwy MA, Goldberg JM, Biscotti CV. Recurrent osseous metaplasia of the cervix after loop electrosurgical excision. Obstet Gynecol 2001; 98: 968-70. [CrossRef]

5. Degani S, Gonen R, de Vries K, Sharf M. Endometrial ossification associated with repeated abortion. Acta Obstet Gynecol Scand 1983; 62: 281-2. [CrossRef]

6. Ejeckam GC, Haseeb F, Ahamad R, Azadeh B. Endometrial ossification. Trop Geogr Med 1991; 43: 314-6.

7. Dorin G, Liliana V, Daniela C, Octavian M, Bianca QG, Cosmin C. Osseous metaplasia of the uterus. TMJ 2003; 53: 280-5.

8. Gal-Gombos EC, Esserman LE, Poniecka AW, Odzer SL, Weisberg S, Godinez J, Poppiti RJ. Osseous metaplasia of the breast: diagnosis with stereotactic core biopsy. Breast J 2002; 8: 50-2. [CrossRef]

9. Sabatini L, Rainey AJ, Tenuwara W, Webb JB. Osseous metaplasia of cervical epithelium. BJOG 2001; 108: 333-4. [CrossRef]

10. Lewis V, Khan-Dawood F, King M, Beckmann C, Dawood MY. Retention of intrauterine fetal bone increases menstrual prostaglandin. Obstet Gynecol 1990; 75: 561-3.

11. Ombelet W. Endoemtrial ossification, an unusual finding in an infertility clinic. A case reported. J Reprod Med 1989; 34: 303-6.

12. Mandato VD, Sacchetti F, Gelli MC, La Sala GB. A hard cervix: Microscopic examination revealed that a rare transformation had occurred. Am J Obstet Gynecol 2012; 206: 362. e1-3. 